temperature remained high and the respiration quick, and the patient complained of some pain in the right side. On Monday (forty hours after the operation) she could breathe through the mouth fairly well, and the silver tracheotomy tube was replaced by a soft rubber one. On Tuesday the tracheotomy tube was dispensed with altogether. As the temperature continued to be high and the respiration quickened the chest was examined, and well marked physical signs of right basal pneumonia were found. On Wednesday (the fourth day after admission and the serenth day after the commencement of the illness) a crisis occurred and the patient made a speedy recovery. There was albumen in the urine for three days only.

Remarks by Dr. THORNTON. - In my limited experience laryngitis of any kind is not usually associated with cases of pneumonia and I have neither heard nor read of any case of oedematous laryngitis necessitating tracheotomy complicating such a condition. That the pneumonia here did not follow the tracheotomy, but was a primary condition, is shown by the onset of the illness, its duration and the evidence of extremely well marked physical signs sixty hours after the tracheotomy was performed, when the chest was examined for the first time.

\section{WOLVERHAMPTON AND STAFFORDSHIRE HOSPITAL.}

A CASE OF PURULENT PERICARDITIS; NECROPSY ; REMARKS (Under the care of Dr. MALET.)

For the account of this case we are indebted to Dr. John Watson, House Physician.

This affection, if estimated by the number of cases on record in the medical journals, is not of very common occurrence in practice, and therefore the account of a case recently seen may be useful pathologically. As this is the second instance of the affection which Dr. Watson has seen he alludes to a few points which appear to be worth mentioning when contrasting the purulent with the ordinary serous form of effusion. In the first place cases of purulent pericarditis are admittedly difficult of certain diagnosis during life. There is not the history of rheumatism to guide the medical man and the disease commences and ends in an insidious manner, the diagnosis belng unfortunately often only made in the post-mortem room. Whether such effusions have any particular pathology of their own or whether they are the result of an ordinary pericarditis becoming purulent from some constitutional peculiarity on the part of the patient is, he considers, a matter of doubt, but it is certain that in both the cases which he has seen the illness was not brought about by rheumatism, tubercle or exposure to damp and wet. It would therefore seem that pericarditis may arise idiopathically, and that when it does so it shows a special tendency towards the purulent form. It appears to be met with more commonly in children than during later life, the subjects in both Dr. Watson's cases being under twelve years of age. The recorded cases also show a heavier incidence upon children than adults. An additional difficulty in regard to diagnosis arises from the fact that there is usually a complete absence of friction sounds over the area affected, owing no doubt to the nature of the effusion, which is much more viscid than is found in ordivary pericarditis. In neither of the cases observed by Dr. Watson was this physical sign present, and in most of those recorded the absence throughout the affection of pericardial friction is particularly emphasised.

A boy aged ten was admitted to the Wolverhampton and Staffordshire Hospital on June 17th last, the mother stating that the lad had been ill for a week, but had been attending school and playing about until the day before admission, when he had complained of nausea, a feeling of chilliness, aching in the legs and loss of appetite, but he had no other symptoms. On examining the chest nothing abnormal could be detected, except that the percussion note was slightly impaired at the right base posteriorly, but air entered the lungs freely in all parts. The heart sounds were, however, almost inaudible and could only be heard as a feeble flutter, no bruit was detected and the apex beat could not be felt. The pulse was 75 ; the temperature was $100^{\circ} \mathrm{F}$. The urine was normal in all respects and the respiration was 20 per minute. He remained in the same condition for the next ten days, when his temperature rose to $103^{\circ} \mathrm{F}$., and he complained of discomfort and slight pain over the præcordium, together with some dyspnœa. The area of cardiac dulness was at this time found to be increased and to extend both laterally towards the left axillary line and downwards towards the epigastrium, the heart sounds and impulse being quite imperceptible. There was a short, hacking cough present for the first time. There was great tenderness on palpating the abdomen (he could hardly bear percussion at all) in the hepatic and epigastric regions, which area was quite dull to percussion and presented a hard, solid feel, being continuous, moreover, with the enlarged area of cardiac dulness above, the one merging imperceptibly into the other. The free edge of the liver could be felt three inches below the free margin of the ribs and extended across the abdomen above the umbilicus. The chest was hyper-resonant all over except the portion before mentioned at the base of the right lung posteriorly and also the upper half of the left chest in front beneath the clavicle. On June 28th dyspnœa was intense, the child had an anxious, pinched expression on his face, the extremities and lips were cyanosed, the alæ nasi working violently, and he was only comfortable when he was in a sitting posture with his shoulders rounded and his arms and head supported on the tray crossing the foot of his cot. On this day some commencing cedema of the subcutaneous structures over the præcordium was observed which pitted on pressure, this continued and increased in severity up to the time of his death. Having seen a similar case some years previously which presented this sign, Dr. Watson was confirmed in his opinion that this was a case of purulent pericarditis when it appeared in the present instance in association with the other symptoms, which from the commencement were typical of pericarditis with effusion. He therefore aspirated the right chest from behind over the seat of the dulness before mentioned and withdrew ten ounces of clear, straw-coloured serum with a few flakes in it, giving great relief to the patient, who remained in much the same condition until July 1st, the fluid in the pleura reaccumulating slowly; but his condition was then so serious that he seemed as though he might die at any moment. His breathing was 48 to the minute, and he was gasping for breath. Some cedema of the lower limbs and genitals was also present, and there was also much cedema of the eyelids and face; his urine contained albumen. As the diagnosis of hepatic abscess had been made in the course of the illness, owing to the manner in which the liver was pushed downwards, it was thought justifiable to try to afford relief by introducing into the liver substance an exploring needle attached to an aspirator, in the hope of tapping a collection of pus. This was done by a member of the staff both from behind and also in front in several places, the needle being moved about when in the liver, but no pus flowed. The patient became progressively worse, the dyspnœa becoming most distressing. The œdema increased, the pulse became intermittent and feeble, and finally death occurred suddenly on July 5th whilst the boy was turning over in bed.

At the post-mortem examination, which was made twentyfour hours after death, the body was found to be very thin and emaciated, the subcutaneous fat being almost entirely absent on the chest walls. On opening the chest the pericardium bulged forwards into the aperture made and was seen to be immensely distended and thickened, and it was firmly adherent to the post-sternal connective tissue. On opening it about three pints of creamy, yellow, pus escaped. The heart itself was lying quite free and projected into the enormously enlarged pericardial cavity, its surface being covered with regetations and lymph, as was also the inner aspect of the whole pericardium. The right lung was adherent to the outer surface of the pericardium in places and was partially collapsed. The left lung was pushed towards the back of the chest cavity and could not be seen at all on first opening the chest, the distended pericardial sac completely hiding it from view. The lung itself was quite collapsed and was almost airless. The liver was enlarged and congested, but the other organs were quite healthy in every respect.

kemarks by Dr. WATson. - There are one or two points which may with advantage be mentioned in connexion with the above case. I have already referred to the absence of the pericardial friction sound throughout, but another symptom which was present in both cases of purulent pericarditis which I have seen was cedema of the præcordial cutaneous structures. I do not remember to have seen it in any case of ordinary serous inflammation of the pericardium, but only in the purulent variety of the disease. The facial œdema and 
also that of the lower extremities and genitals are accounted for by the pressure exercised by the distended pericardium upon the large vessels in the thorax. The effusion into the pleura can be similarly explained, as well as the cyanosis and lividity of the extremities. The serum in the pleural cavity showed an immediate tendency to reaccumulate in spite of repeated tapping. The downward displacement of the liver is very misleading, as in this case it was thought by some that there was an accumulation of pus in that organ or between it and the diaphragm, and it was with this idea that exploring needles were introduced into the liver both from behind and in front. Other diagnoses which were proposed were pleural effusion, empyema, enlarged glands in the posterior mediastinum and a growth in the mediastinum. Lastly, the temperature was anytbing but a help in the matter of diagnosis. On admission it was $100^{\circ}$ and remained below $1016^{\circ}$ until the sudden rise to $103^{\circ}$ on the evening of June 27th. It varied between normal and $100^{\circ}$ until July 2 nd, when it became normal and remained so until death occurred.

\section{COUNTY LUNATIC ASYLUM, LANCASTER.}

A CASE OF ACROMEGALY.

MANY cases of this disease have been described since Dr. Marie first published an account of two patients under the care of Professor Charcot in 1886 . It is, however, sufficiently rare to make the record of a case under careful observation of some interest, as thus we can compare it with others and note the absence or presence of symptoms regarded as important. It was supposed that certain pathological changes besides those of the skeleton and connective tissue were constantly found-viz., (1) simple hypertrophy of the pituitary body, with dilatation of the sella turcica; (2) the persistence of the thymus gland; and (3) the hypertrophy of the fibres and ganglia of the sympathetic system-but subsequent necropsies have proved that these changes are not invariably present. Dr. Fräntzel found no thymus gland and the pituitary gland was apparently normal. Dr. Waldo found cystic cavities in the brain, but the pituitary body was normal. Dr. Gauthier doubts the influence of changes in the pituitary body in the production of the disease. On the other hand, Dr. Marie regards changes in the pituitary body as important, and speaks of it as "almost invariably much enlarged."

Dr. Holsti found it very much enlarged in a case described by him. For the account of this case we are indebted to Mr. S. Allan Shiach, the superintendent pathologist and assistant medical officer.

A married woman, thirty-six years of age, was admitted to the Lancaster County Lunatic Asylum on May 1st, 1891, when she was found to be an unsightly looking woman, with a low forehead, coarse hair, very large nose and large hands and feet. Her speech was slow and her general health was good. She had had four children, of whom one is alive. She had been of intemperate habits and had suffered from severe headaches for six months previously to admission. There was no history of specific disease. As regarded her mental condition, she was subject to melancholia with delusions.

In June last the forehead was slightly retreating and contracted, the anterior parts of the temporal bones being depressed. With the exception of some thickening about the middle of the sagittal suture there were no crests to be found in the lines of the cranial sutures. The external occipital protuberance with its ridges and the mastoid processes were not affected. There was a projection round both orbits due to a thickening of the superciliary ridges, the skin over which was also thickened. The eyelids were slightly longer but not thicker than normal. The whole face was elongated and was of an oval shape; the nose was greatly increased in size and formed a marked projection; the septum and alæ nasi were thickened to more than twice their normal size ; the malar bones were hypertrophied; both lips were hypertrophied, the lower one being everted; the lower jaw was markedly prognathous in type and when the two jaws were closed it was in front of the upper one; the angle of the lower jaw was an extremely oblique one; the tongue was enlarged and flabby ; there were only a few teeth in the upper jaw and many of those in the lower jaw were carious; the uvula was increased in size; there was no departure from the normal in the curvatures of the spine; the sternum was thickened and so were the ribs, but to a slight extent only; the clavicles were increased in size, especially at their sternal extremities; there was no change in the scapula or its processes, in the vertebra or in the pelvic bones; both hands were markedly acromegalic in type and the term "spade-like" could be well used in describing them; the fingers were short and stumpy and the proximal and distal phanlanges were equally bypertrophied. The thenar and hypothenar eminences were increased in thickness. The nails were longitudinally striated. The bones of the forearm and arm were not enlarged. The feet were affected similarly to the hands, but not to such a marked degree. The lower ends of the tibia and fibula were thickened. The larynx was slightly thickened and the thyroid cartilage was more prominent than normal, but the thyroid body did not appear to be affected. In the upper sternal region the dulness due to the remains of the thymus gland and mentioned by Schültze and Verstraeten, was looked for but without result. The area of heart dulness was increased, but there was no bruit present. The arteries and veins were healthy. Perspiration was a marked feature in this case and it appeared with the slightest exertion ; it was very profuse in amount and it had a most disagreeable odour. There was no albumen, sugar or peptones present in the urine. The susceptibility to cold was increased and the circulation in the extremities was very poor. Severe pain was very frequently felt in the head, occurring during both the day and the night. The pain was referred to the vertex of the cranium, about the middle of the sagittal suture. No pains were felt in the joints or the limbs. Menstruation had not taken place for a year, and for six years before that it had been very irregular. None of the special senses seemed to be affected. The appetite was good, and when headache was present a full meal often succeeded in driving it away or in alleviating the pain. Her wedding ring had required alteration twice owing to the increase in the size of her finger, but for the last year there had been hardly any change. The voice was harsh and grating.

Remarks. - The objective phenomena in this case are very noticeable and seem to indicate unmistakably that the diagnosis of acromegaly is a correct one. The symmetrical enlargement of the hands and feet, the prognathism of the lower jaw and the hypertrophy of the lips \&c. are present. Pierre Marie, who first described this disease and who named it, says that curvatures of the spine in the cervicodorsal region and disturbances of vision are nearly always seen in cases of acromegaly. The patient in the present case does not present these symptoms, but may probably do so on further development.

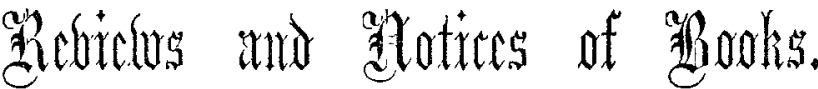

Outines of the Diseases of Tomen. By JoHn Phillips, M. A., M.D., Assistant Obstetric Physician to King's College Hospital. With 120 Illustrations. London: Charles Griffin and Co., Limited. 1893.

IN preparing a text-book intended, as this is, "entirely for the use of the student and junior practitionor," it is essential. that the author should do as he has done, and begin at the very beginning with the most elementary part of the subject; for, as he says, no scientific knowledge of it "can be attained without a close attention to first principles." A book based on these lines, on such a subject as gynæcology, must necessarily contain much that has been previously ascertained by a multitude of workers in various parts of the field, and an author's opportunity of displaying originality is therefore considerably restricted. He can, however, show a sound judgment in selecting what seems to him best from standard works on the subject and he can incorporate with this any comments or original observations, as occasion may arise, that are suggested by his own experience. In the preface Dr. Phillips very rightly insists on the importance of an accurate acquaintance with the female pelvic organs for the proper study of the diseases of women. The student. must have a clear understanding as to what is normal before he can profitably study what is pathological. As regards: Fig. 1, an outline drawing of the female pudendum, we think it would be almost unintelligible to a beginner. For instance, a continuous line, that might equally represent the outline of a belmet or a bell, is intendcd 